\title{
Bouveret's syndrome: a case report and a review of the literature
}

\author{
Woohyung Lee ${ }^{1}$, Sung-Sik Han², Seung Duk Lee ${ }^{2}$, Young-Kyu Kim², Seong Hoon Kim², \\ Sang Myung $\mathrm{Woo}^{2}$, Woo Jin Lee ${ }^{2}$, Young Whan $\mathrm{Koh}^{2}$, Eun Kyung $\mathrm{Hong}^{2}$, and Sang-Jae Park ${ }^{2}$ \\ ${ }^{1}$ Department of Surgery, Seoul National University College of Medicine, Seoul, \\ ${ }^{2}$ Center for Liver Cancer, National Cancer Center, Goyang, Korea
}

\begin{abstract}
Bouveret's syndrome is a gastric outlet obstruction caused by an impacted gallstone that passes through a cholecysto-gastric or cholecysto-duodenal fistula. An elderly woman visited a local clinic with nausea and abdominal pain. Abdominal computed tomography revealed a stone that was impacted in the duodenal lumen and a fistula between the gallbladder and duodenum. Malignancy could not be excluded due to the mass in the cystic duct showing enhancement and the presence of enlarged lymph nodes on computed tomography, and increased fludeoxyglucose uptake in the cystic duct on positron emission tomography. The patient underwent simultaneous cholecystectomy, segmental duodenectomy and gastro-jejunostomy. Pathological examination exhibited chronic inflammation and no primary cancer of the gallbladder and fistula. (Korean J Hepatobiliary Pancreat Surg 2012;16:84-87)
\end{abstract}

Key Words: Bouveret's syndrome; Cholecysto-duodenal fistula; Gallstone ileus

\section{INTRODUCTION}

The common causes of gastric outlet obstruction include peptic ulcer disease, stomach cancer and gastrointestinal stromal tumor. Impacted gallstone is a rare cause of intestinal obstruction and accounts for $1 \%$ to $3 \%$ of all intestinal obstructions. ${ }^{1,2}$ Bouveret's syndrome is a gastric outlet obstruction caused by an impacted gallstone that passes through a cholecysto-gastric or cholecysto-duodenal fistula. ${ }^{3,4}$ We present a case of Bouveret's syndrome which was successfully treated with endoscopic stone removal, followed by cholecystectomy, resection of the duodenal bulb and the distal stomach and gastro-jejunostomy.

\section{CASE}

A 76-year-old woman visited a local clinic with a history of nausea and abdominal pain. She did not have a specific past medical history except hypertension. A clinician found gastritis on endoscopy and a stone in the gall- bladder on ultrasonography. To evaluate the gallstone, she was transferred to our hospital.

On physical examination, the patient had tenderness in the right upper quadrant. Her bowel sounds were normoactive. Laboratory tests, including blood cell counts, liver enzymes, electrolytes and creatinine levels were within normal limits. Abdominal computed tomography (CT) revealed a distended stomach, an irregular wall thickening at the first and second portions of the duodenum and a gallbladder with an enhancing mass in the cystic duct (Fig. 1). There was a cholecysto-duodenal fistula with a $3-\mathrm{cm}$ calcified stone in the duodenal lumen. Enlarged lymph nodes were seen along the common hepatic artery. Magnetic resonance cholangiopancreatography revealed a mild intrahepatic duct dilatation and a mild narrowing of the confluence. Positron emission tomography (PET) showed a slight increase in fludeoxyglucose uptake $(\mathrm{SUV}=3.9)$ in the cystic duct, which made it impossible to rule out the possibility of gallbladder cancer (Fig. 2).

Esophagogastroduodenoscopy (EGD) showed a $2.3 \times$ $2.8-\mathrm{cm}$ pigment stone causing gastric outlet obstruction.

Received: March 25, 2012; Revised: April 30, 2012; Accepted: May 3, 2012

Corresponding author: Sung-Sik Han

Center for Liver Cancer, National Cancer Center, 323, Ilsan-ro, Ilsandong-gu, Goyang 410-769, Korea

Tel: +82-31-920-1641, Fax: +82-31-920-1138, E-mail: sshan@ncc.re.kr 


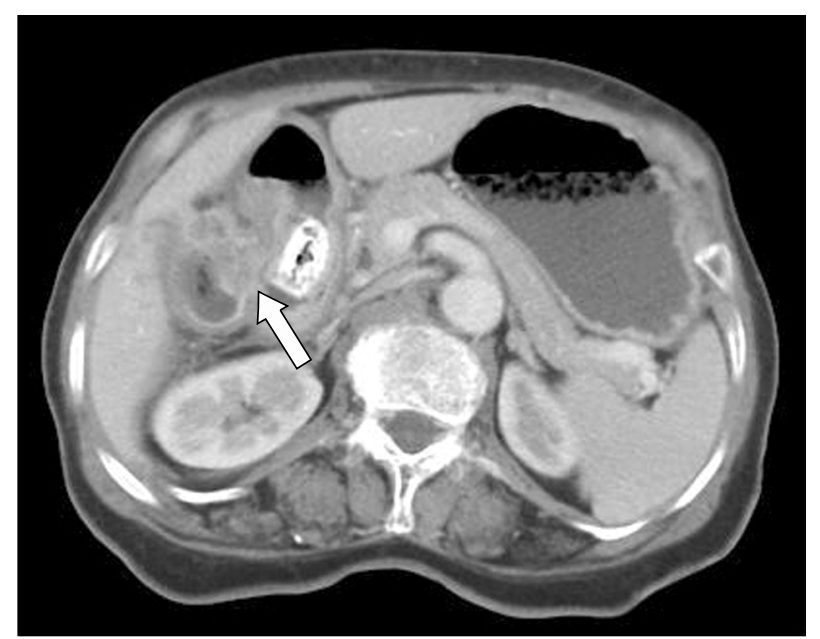

Fig. 1. CT shows a cholecysto-duodenal fistula (arrow) and an impacted gallstone in the duodenum.

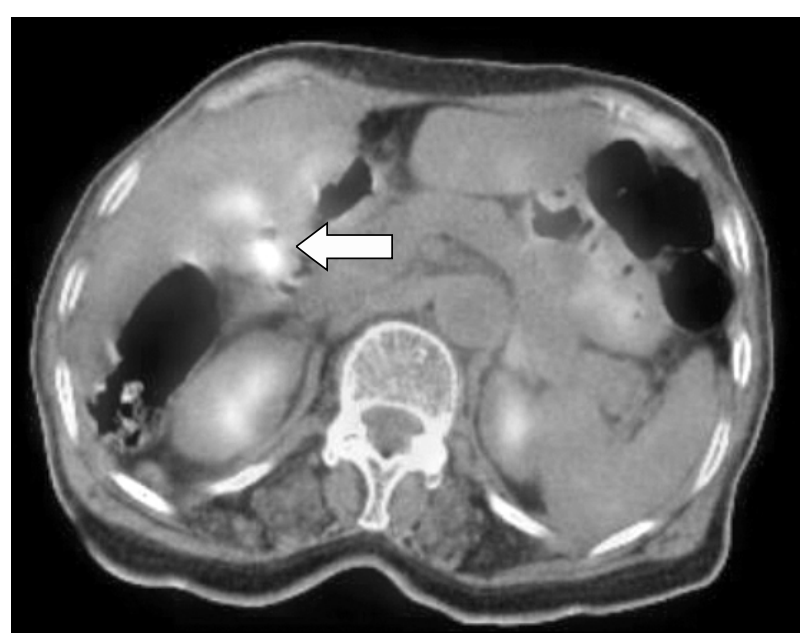

Fig. 2. PET shows increased FDG uptake $(\mathrm{SUV}=3.9)$ in the cystic duct (arrow).
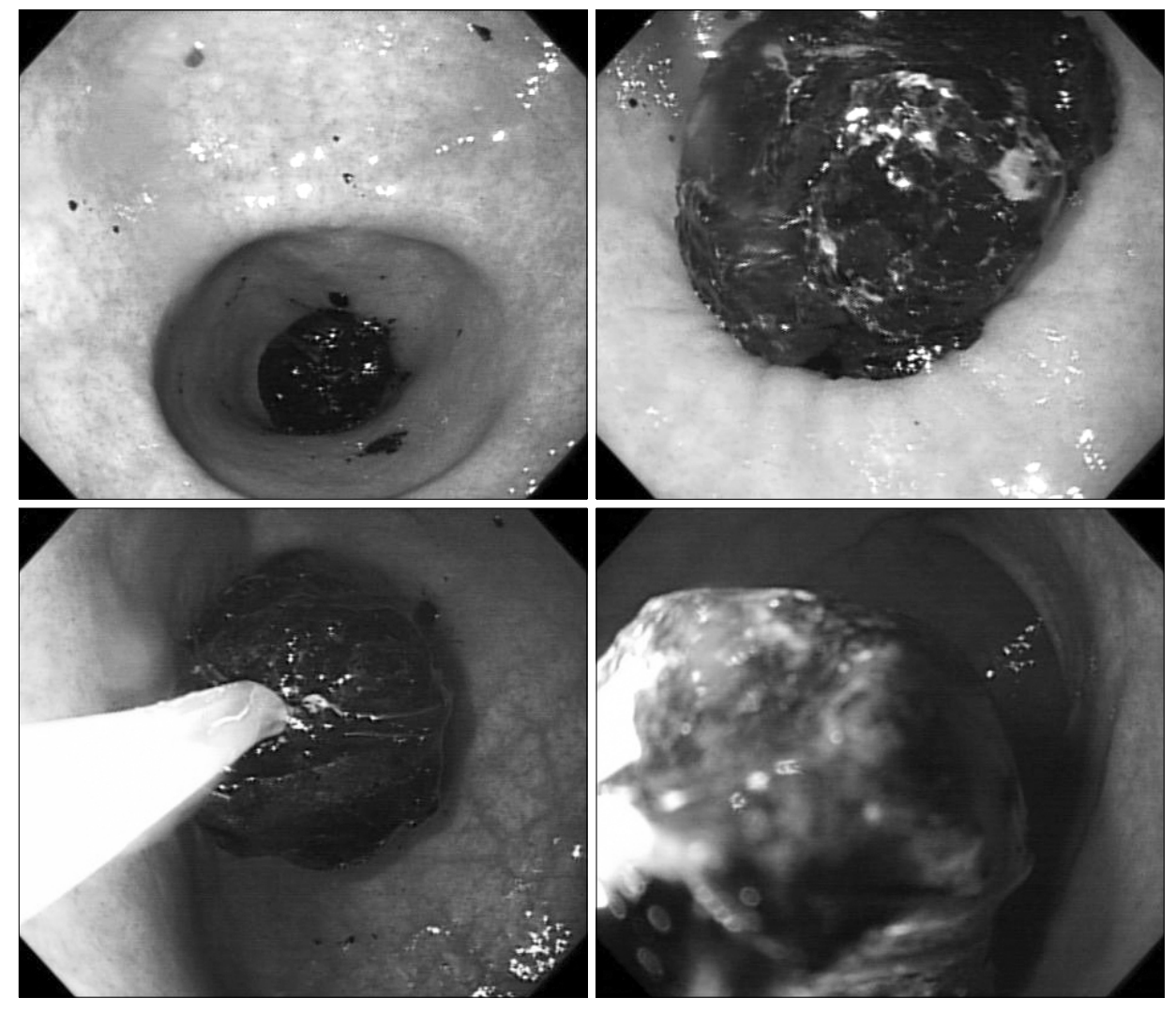

Fig. 3. EGD shows an intraluminal stone in the duodenal bulb. The stone was removed using an extracting basket.
The stone was successfully removed from the duodenum using an extracting basket (Fig. 3). A severe ulceration was observed on the duodenal wall where the gallstone was impacted. However, a cholecysto-duodenal fistula was not observed on EGD due to the duodenal deformity. One week later, the patient underwent operation.

In the operative field, the gallbladder showed severe in- flammation and adhesions to the duodenum, which had resulted in a cholecysto-duodenal fistula. After cholecystectomy, we resected the duodenal bulb along with the distal stomach and also performed gastro-jejunostomy. The patient started her diet on postoperative day 4. A percutaneous drainage tube was inserted into the anterior aspect of the stomach for removal of fluid accumulation on post- 
operative day 6. She was discharged on postoperative day 22 after the drainage tube was extubated. Histopathological examination revealed chronic cholecystitis with no evidence of malignancy.

\section{DISCUSSION}

In 1896, Bouveret reported 2 patients with gastric outlet obstruction due to an impacted gallstone. ${ }^{1}$ Recurrent inflammation of the gallbladder frequently causes erosion and necrosis of the gallbladder wall and forms biliary-enteric fistulas. There are many types of fistulas according to the site of fistula formation, such as cholecysto-gastic, cholecysto-duodenal or cholecysto-colic fistula. ${ }^{3,4}$ Fistulas are known to occur twice more frequently in women than in men. ${ }^{5}$

The symptoms of Bouveret's syndrome are not typical. Nausea/vomiting is the most common symptom, followed by abdominal pain, abdominal distension, hematemesis, weight loss and melena. Abdominal tenderness is commonly noted on physical examination. ${ }^{4}$

Pneumobilia, a calcified mass or a distended stomach is frequently seen in plain abdominal radiographs. Ultrasonography can detect cholecysto-duodenal fistulas. However, if a fistula tract is filled with fluid or air, it may be confused with the common bile duct. CT is helpful in detecting pneumobilia, cholecysto-duodenal fistula and distended stomach. Magnetic resonance cholangiopancreatography is a diagnostic tool that provides definitive imaging of cholecysto-duodenal fistulas. T-2 weighted magnetic resonance imaging shows intraluminal duodenal filling defects and cholecysto-duodenal fistulas. ${ }^{6-8}$

EGD has diagnostic and therapeutic values. Impacted stones can be removed through EGD using a stone basket. Intracorporeal laser lithotripsy, electrohydraulic lithotripsy and extracorporeal shock wave lithotripsy as well as a manual endoscopic lithotripsy have recently been performed to treat patients with gallstones. ${ }^{9,10}$ Even after successful endoscopic stone removal, surgery must be considered when there is biliary obstruction, remnant gallstone, cholecystitis or recurrent cholangitis. Surgical treatments include enterolithotomy, cholecystectomy and fistula repair. There are 2 surgical options. An one-stage operation is conducted to perform enterolithotomy, cholecystectomy and fistula repair simultaneously. A two-stage operation is considered when patients' vital signs are unstable and consists of an enterolithotomy first and then delayed cholecystectomy with fistula repair. ${ }^{11-17}$ The mortality rate is reported to be higher in the one-stage operation than in the two-stage operation $(20-30 \%$ vs. $12 \%) .{ }^{13}$ However, biliary complications are more frequent after enterolithotomy alone. ${ }^{15}$ Therefore, therapeutic plans should be made based on the patient's general condition. Surgical procedures are variable depending on the site and size of fistulas. If a fistula opening is small, primary repair can be attempted. Otherwise, gastric or duodenal resection and gastro-enteric anastomosis should be performed. ${ }^{11-13}$

Chronic inflammation of the gallbladder caused by cholecysto-enteric fistulas may induce malignant transformation. Singer et al. ${ }^{18}$ and Sanada et al. ${ }^{19}$ reported some cases of malignant transformation of Bouveret's syndrome. Day and Marks ${ }^{20}$ reported 2 cases of biliary-enteric fistulas associated with gallbladder cancer in 34 patients with gallstone ileus. In our case, malignancy was suspected because of the findings of an enhanced mass with increased fludeoxyglucose uptake in the cystic duct. However, there was no evidence of malignancy on histopathological examination.

\section{REFERENCES}

1. Puri V, Lee RW, Amirlak BA, et al. Bouveret syndrome and gallstone ileus. Surg Laparosc Endosc Percutan Tech 2007;17:328330 .

2. O'Neill C, Colquhoun P, Schlachta CM, et al. Gastric outlet obstruction secondary to biliary calculi: 2 cases of Bouveret syndrome. Can J Surg 2009;52:E16-18.

3. Iancu C, Bodea R, Al Hajjar N, et al. Bouveret syndrome associated with acute gangrenous cholecystitis. J Gastrointestin Liver Dis 2008; 17:87-90.

4. Cappell MS, Davis M. Characterization of Bouveret's syndrome: a comprehensive review of 128 cases. Am J Gastroenterol 2006;101:2139-2146.

5. Doycheva I, Limaye A, Suman A, et al. Bouveret's syndrome: case report and review of the literature. Gastroenterol Res Pract 2009;2009:914-951.

6. Pickhardt PJ, Friedland JA, Hruza DS, et al. Case report. CT, MR cholangiopancreatography, and endoscopy findings in Bouveret's syndrome. AJR Am J Roentgenol 2003;180:1033-1035.

7. Singh AK, Shirkhoda A, Lal N, et al. Bouveret's syndrome: appearance on $\mathrm{CT}$ and upper gastrointestinal radiography before and after stone obturation. AJR Am J Roentgenol 2003;181:828-830.

8. Brennan GB, Rosenberg RD, Arora S. Bouveret syndrome. Radiographics 2004;24:1171-1175.

9. Tanwar S, Mawas A, Tutton M, et al. Successful endoscopic management of Bouveret's syndrome in a patient with cholecystoduodenocolic fistulae. Case Rep Gastroenterol 2008;2:346350 . 
10. Alsolaiman MM, Reitz C, Nawras AT, et al. Bouveret's syndrome complicated by distal gallstone ileus after laser lithotropsy using Holmium: YAG laser. BMC Gastroenterol 2002;2:15.

11. Mittal S, Sutcliffe RP, Rohatgi A, et al. A possible variant of Bouveret's syndrome presenting as a duodenal stump obstruction by a gallstone after Roux-en-Y gastrectomy: a case report. J Med Case Rep 2009;3:7301.

12. Rodríguez-Sanjuán JC, Casado F, Fernández MJ, et al. Cholecystectomy and fistula closure versus enterolithotomy alone in gallstone ileus. Br J Surg 1997;84:634-637.

13. Erlandson MD, Kim AW, Richter HM 3rd, et al. Roux-en-Y duodenojejunostomy in the treatment of Bouveret syndrome. South Med J 2009;102:963-965.

14. Chowbey PK, Bandyopadhyay SK, Sharma A, et al. Laparoscopic management of cholecystoenteric fistulas. J Laparoendosc Adv Surg Tech A 2006;16:467-472.

15. Sica GS, Sileri P, Gaspari AL. Laparoscopic treatment of Bouveret's syndrome presenting as acute pancreatitis. JSLS 2005;9:472-
475 .

16. Arioli D, Venturini I, Masetti M, et al. Intermittent gastric outlet obstruction due to a gallstone migrated through a cholecysto-gastric fistula: a new variant of "Bouveret's syndrome". World J Gastroenterol 2008;14:125-128.

17. Kavuturu S, Parithivel V, Cosgrove J. Bouveret's syndrome: a rare presentation of gallstone disease. OPUS 12 Scientist 2008; 2:25-26.

18. Singer M, Chandar VP, Hookman P. Gallbladder carcinoma with gastric outlet obstruction and gallstone ileus. South Med J 1986; 79:902-903.

19. Sanada T, Baba H, Ohba A, et al. Gallbladder carcinoma, progressed along cholecystoduodenal fistula--a case report. Gan To Kagaku Ryoho 2010;37:2717-2719.

20. Day EA, Marks C. Gallstone ileus. Review of the literature and presentation of thirty-four new cases. Am J Surg 1975;129: $552-558$. 